\title{
Food-anticipatory response to restricted food access based on the pigeon's biological clock
}

\author{
HIROSHI ABE \\ Nagoya University, Nagoya, Japan \\ and \\ SUKEO SUGIMOTO \\ Hiroshima University, Hiroshima, Japan
}

\begin{abstract}
Pecking at the food key was recorded for 4 pigeons given restricted access to food. The access period was set at a fixed time in a light-dark cycle, continuous dark, or continuous light. The pecking activity occurred a few hours before onset of the access period in all three conditions. When the bird was again given free access to food after being released from restricted access, its pecking rhythm free-ran in the continuous dark. The initial phase of the rhythm coincided with the onset of the food-anticipatory pecking in the previous condition. These resulte suggest that the bird anticipated food access, based on its biological clock mechanism. When the access period was set in the dark phase of the light-dark cycle, anticipatory pecking did not occur, although pecking actually occurred during the access period. The pigeon's activity is reduced during the dark phase of the light-dark cycle. Therefore, the bird's activity level was probably too low to shape the anticipatory response, even if the access period was stored in memory in the biological clock.
\end{abstract}

When a rat is repeatedly presented with food at a fixed time of day (restricted food access), the animal shows an increased level of wheel-running for a certain period preceding the onset of the access period. This increased activity was first reported by Richter (1927) and was attributed to the anticipation of food. This suggests that the rat can learn the access time based either on the temporally conditioned state of motivation or on the internal clock mechanism.

Bolles and deLorge (1962) and Bolles and Stokes (1965) later showed that food-anticipatory activity occurred only when an animal was presented with food every $24 \mathrm{~h}$ (circadian), and was not observed when the food was delivered every 19 or $29 \mathrm{~h}$ (noncircadian). This finding strongly favors the possibility that learning and memory of the access period are based on the biological clock, not on the temporal conditioning of the motivational state. Similar results and suggestions have been reported by other researchers (Boulos, Rosenwasser, \& Terman, 1980; Edmonds \& Adler, 1977; Rosenwasser, Pelchat, \& Adler, 1984; Stephan, Swann, \& Sisk, 1979a).

Whereas many studies on restricted food access have been conducted with the rat (Boulos \& Terman, 1980; Terman, Gibbon, Fairhurst, \& Waring, 1984), only a few

The authors would like to thank K. Tsuji, T. Mano, and M. Kida, Nagoya University, for their careful editing and comments on the manuscript. S. Sugimoto is with the Department of Behavioral Sciences, Hiroshima University. Requests for reprints should be sent to H. Abe, who is now with the Department of Aerospace Psychology, Research Institute of Environmental Medicine, Nagoya University, Furo-cho, Chikusa-ku, Nagoya 464, Japan. studies have reported results with avian species. Starlings (Adler, 1963a, 1963b) and several other species of birds (Stein, 1951) were observed under restricted food access in connection with navigation and seasonal migration. These birds were found to have memory of the access period. However, the pigeon has not so far been observed, despite its being a commonly used laboratory animal in behavioral studies.

The present study examined whether food-anticipatory activity depended on the biological clock in the case of the pigeon, as in other species. The effect of a shift in the light-dark regime nonconcurrent with the food access period was also tested.

\section{METHOD}

\section{Subjects}

Four male homing pigeons (Columba livia) were observed. They were about 4 years old and weighed $332-392 \mathrm{~g}$ at the start of the experiment.

\section{Apparatus}

Bird P821's home cage measured $45 \times 45 \times 50 \mathrm{~cm}$; the other 3 birds' cages measured $45 \times 45 \times 70 \mathrm{~cm}$. The cages were placed in individual chambers. Each chamber was equipped with a ventilating fan, which successfully masked noises from outside. Lighting inside the chamber was supplied by two fluorescent lights (4 W each), giving $300 \mathrm{~lx}$ during the light period and $0.1 \mathrm{~lx}$ during the dark period at the center of the chamber floor. Ambient temperature was not controlled strictly, but was kept at $15^{\circ}-25^{\circ} \mathrm{C}$.

The cage was equipped with a pecking key and a food tray on a side wall. The key was dimly illuminated from behind when necessary (the intensity of key illumination was $0.03 \mathrm{~lx}$ at the center of the chamber). When a bird pecked at the key 10 times, the food 
tray was raised and the bird could get access to food (mixed grains) for $3 \mathrm{sec}$ (fixed-ratio reinforcement schedule: FR10). But for Bird P821, the food was given when the bird pecked at the key $5 \mathrm{sec}$ after the previous reinforcement (fixed-interval reinforcement schedule: FI $5 \mathrm{sec}$. The reinforcement schedule was controlled by computers (NEC TK-80/BS, OKI IF-800). The pecking response was recorded on an event recorder and accumulated at 5-min intervals by a computer (OKI IF-800). Data fed into the computer were digitalized for 5-min bins. If any peck occurred in one bin, the bin was indicated with a vertical strike on the time scale. To make visual inspection of the free-running of the rhythm easier, the data were double-plotted.

Water was available at all times in the cage. Water was replaced every day and food was renewed every 3 days. The cage was cleaned every day. This work was done at different times of day.

\section{Procedure}

Fach of the 4 birds was observed under various conditions. Conditions and their sequence differed among birds. Conditions were as follows: (1) FF-LD-Free food access (FF) under a 12-h lightdark (LD) cycle (light period from 0600 to 1800). (2) FF-DDFree food access under continuous dark (DD). (3) RF-LDRestricted food access (RF) under a 12-h light-dark cycle. The food access period was restricted for $2 \mathrm{~h}$ starting at a fixed time in the light period of the cycle. (4) RF-DD-Restricted food access un- der continuous dark. The $2-h$ food access period was set in the dark period at a fixed time of day. (5) $R F-L L-$ Restricted food access under continuous light (LL). The 2-h food access period was set in the light period at a fixed time of day. (6) RF-DL-Restricted food access under a 12-h dark-light cycle (DL) (light from 1800 to 0600 ). The 2 -h food access period was set at a fixed time of day in the dark period of the cycle.

In the FF sessions, the pecking key was always dimly illuminated. However, for the first 10 days of the first RF session, the key was dimly illuminated only during the food access period in order to give an external cue for food availability. Then the cue was eliminated by giving continuous illumination of the key again. The birds thus could not identify the food access period unless they actually pecked at the key during the period. Each session continued for at least 20 days.

\section{RESULTS}

In the FF condition, the pecking rhythm was entrained to the LD cycle (FF-LD). Pecking occurred and extended mainly over the light period of the cycle, as is shown in Figures 1 and 2 (see Days 1-15 in Figure 1 and Days 1-23 in Figure 2). The pecking rhythm free-ran in FF-DD.

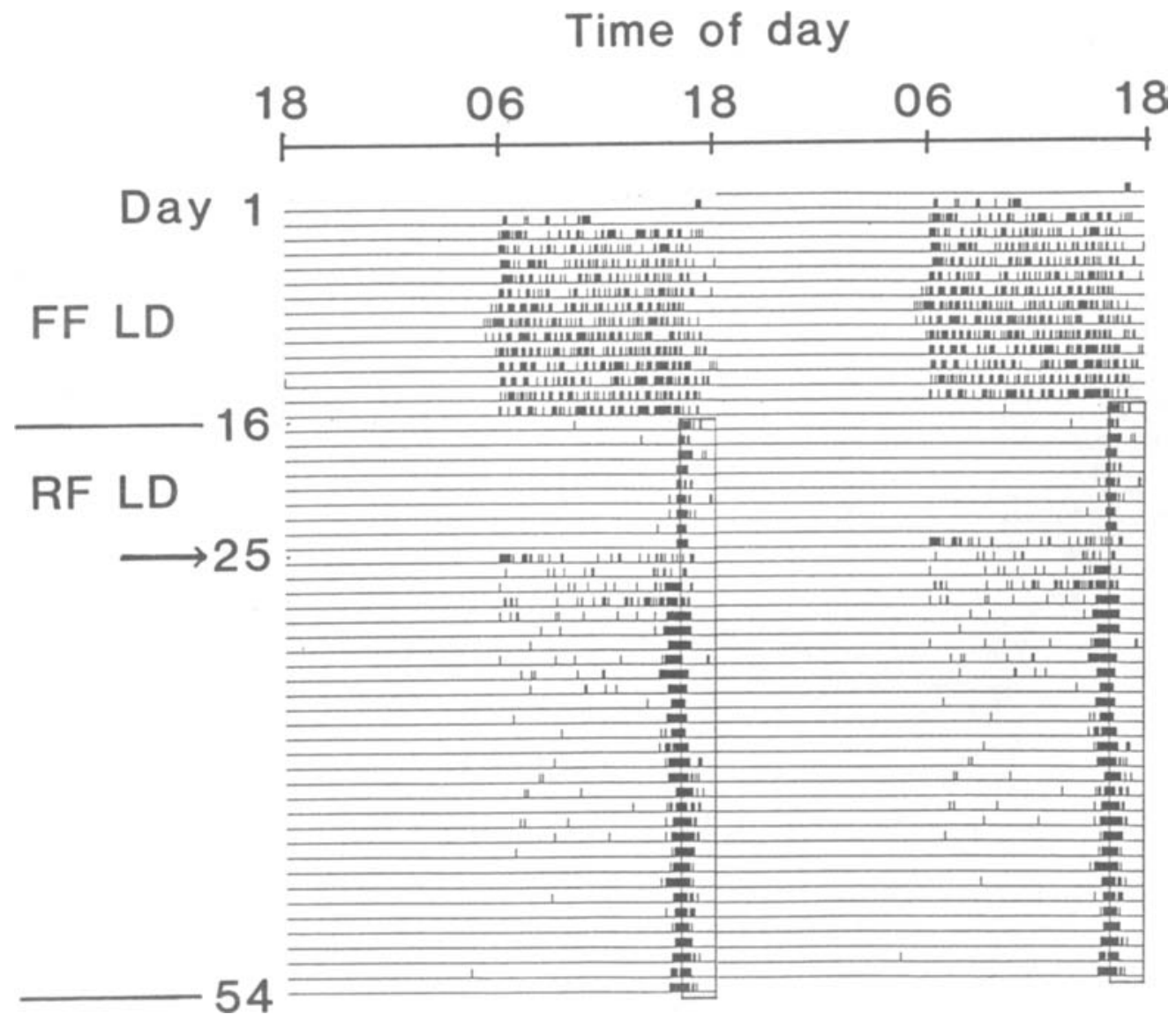

Figure 1. Temporal distribution of pecking responses under light-dark (LD) 12:12 (light period: 0600-1800) for Bird P834. Data are double-plotted. The area enclosed with a solid line indicates the food access period. From Day 16 to Day 24 the pecking key was dimly illuminated only during the food access period, and from Day 25 onward the key light was on continuously. RF $=$ restricted food access; FF $=$ free food access. 


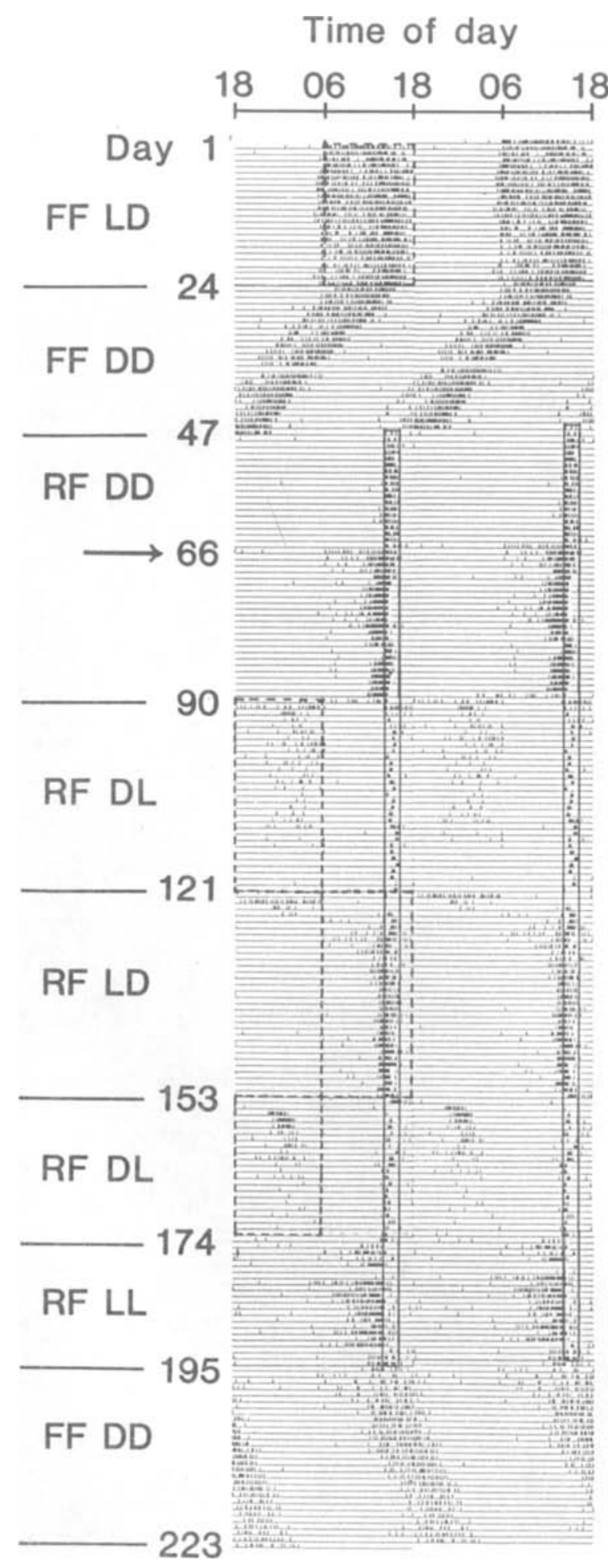

Figure 2. Temporal distribution of pecking responses for Bird P831. The area inside the broken square indicates the light period of the light-dark (LD) cycle. The area enclosed with a solid line indicates the food access period. From Day 47 to Day 65 the key was illuminated only for the restricted food access period; from Day 66 forward, the key was lit continuously. FF = free food access; $\mathbf{R F}=$ restricted food access; $D D=$ continuous dark; $L L=$ continuous light; DL $=$ dark-light cycle (light period: $1800-0600)$.
This free-running period was shorter than $24 \mathrm{~h}$, as is shown in Figures 2 and 3 (see Days 24-46 in Figure 2 and Days 1-16 in Figure 3). However, when the food access period was restricted for $2 \mathrm{~h}$ at a fixed time of day under DD (RF-DD), a free-running rhythm was not seen. When a key was illuminated during the food access period as an external cue, pecking occurred only during that period (Days 47-65 in Figure 2 and Days 17-30 in Figure 3). After the cue was eliminated, pecking occurred during the period except for the access period (see Days 66-89 in Figure 2 and Days 31-66 in Figure 3). Pecking occurred about $12 \mathrm{~h}$ before the onset of the access period, but gradually became restricted for 1-2 $\mathrm{h}$ before the onset of the access period. This bout of pecking activity is regarded as the anticipation of food.

The food-anticipatory activity showed the phenomenon similar to "relative coordination" that usually occurred when the rhythm was entrained to the LD cycle. For Bird P831 (see Figure 2), the activity onset gradually advanced between Days 66 and 80 , and then delayed until it was timed just at the access period on Days 81-83. Then the onset of the activity advanced again from Day 84 to 89. This phenomenon was also seen in Bird P833 (Figure 3, from Day 31 on).

The anticipatory activity was observed in RF-LL as well (see Days 174-194 in Figure 2), although it was not so clear as that observed in RF-DD, since pecking was distributed diffusely. The activity onset was 6 to $10 \mathrm{~h}$ before the onset of the access period.

When a bird was released from restricted food access and given free access (FF-DD), pecking activity free-ran (see Days 195-223 in Figure 2 and Days 47-90 in Figure 4). The initial phase of free-running rhythm coincided with the phase of anticipatory activity in the previous RF session. The free-running period was not constant. Bird P831 showed a period that was shorter than $24 \mathrm{~h}$ before the RF session (Days 24-46, Figure 2) but longer than $24 \mathrm{~h}$ after the session (Days 195-223, Figure 2). Bird P821 showed a period shorter than $24 \mathrm{~h}$ after the session (Days 47-90, Figure 4), although the period of this bird tended to fluctuate.

The anticipatory activity was also observed in RF-LD. The food access period was set in the light period. When the key illumination was restricted to the food access period, pecking occurred only during that period (see Days 16-24 in Figure 1). However, after the key was illuminated continuously, pecking occurred beginning $12 \mathrm{~h}$ before the access period (Days 25-28, Figure 1). The pecking activity gradually became concentrated within 1 or $2 \mathrm{~h}$ before the access period. Similar activity was observed in RF-LD for Bird P831 (see Days 121-152 in Figure 2). In this case, an LD session was inserted between two DL sessions. Pecking began prior to the food access period and became concentrated within 1 or $2 \mathrm{~h}$ before the onset of the access period. The foodanticipatory activity was thus formed and maintained during the session.

In RF-DL, the food access period was set in the dark period of the LD cycle. The bird showed pecking activity 


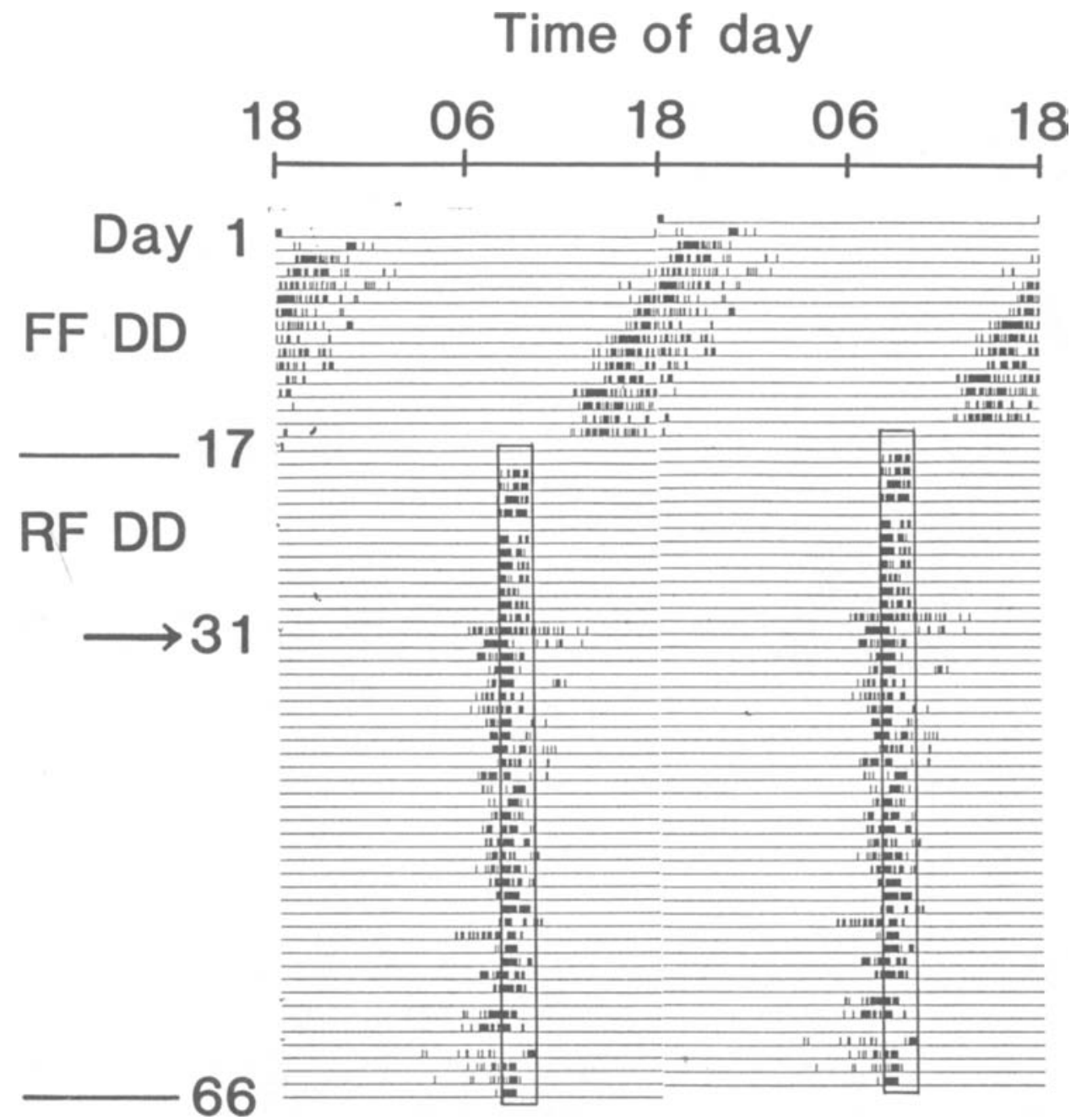

Figure 3. Temporal distribution of pecking responses under continuous dark (DD) for Bird P833. The area enclosed with a solid line indicates the food access period. From Day 17 to Day 30 the pecking key was dimly illuminated only during the food access period; from Day 31 onward the key was illuminated continuously. $\mathrm{FF}=$ free food access; $\mathrm{RF}=$ restricted food access.

during the access period but anticipatory pecking was not observed, as is clearly shown in Figure 2 (see Days 90-120). This was confirmed by the fact that the anticipatory pecking disappeared after the lighting condition was shifted from LD to DL (see Days 153-173 in Figure 2).

\section{DISCUSSION}

Anticipatory pecking activity was observed in the pigeon under conditions of restricted food access. The anticipatory pecking occurred not only in LD but also in DD and $L L$.

Anticipatory pecking was gradually formed after the key was illuminated continuously. The bird could not know when to expect the food access without pecking a key. On the first 1 or 2 days after the cue was eliminated, pecking was distributed over a wide range of time. Afterward, however, pecking became more sharply concentrated dur- ing 1-2 $\mathrm{h}$ before the onset of the access period. These results indicate that the bird learned the food access period, which was set at a fixed time of day, and retained it in memory. The results for RF-DD and RF-LL suggest that this learning and memory of the food access period depend on temporal information acquired by the pigeon on the basis of its internal biological clock.

This possibility might also be supported by an observation in FF-DD session after the bird was released from restricted access: The onset of the free-running rhythm was at the same time as that of the anticipatory activity in the previous RF session. This result suggests that the pecking activity was entrained to the periodic feeding $\mathrm{cy}$ cle regulated by the biological clock.

Memory of the food access time has been reported in honeybees (Renner, 1960). When the honey tray was placed on a fixed point at a fixed time of day, the honeybees learned not only the place but also the time that honey 


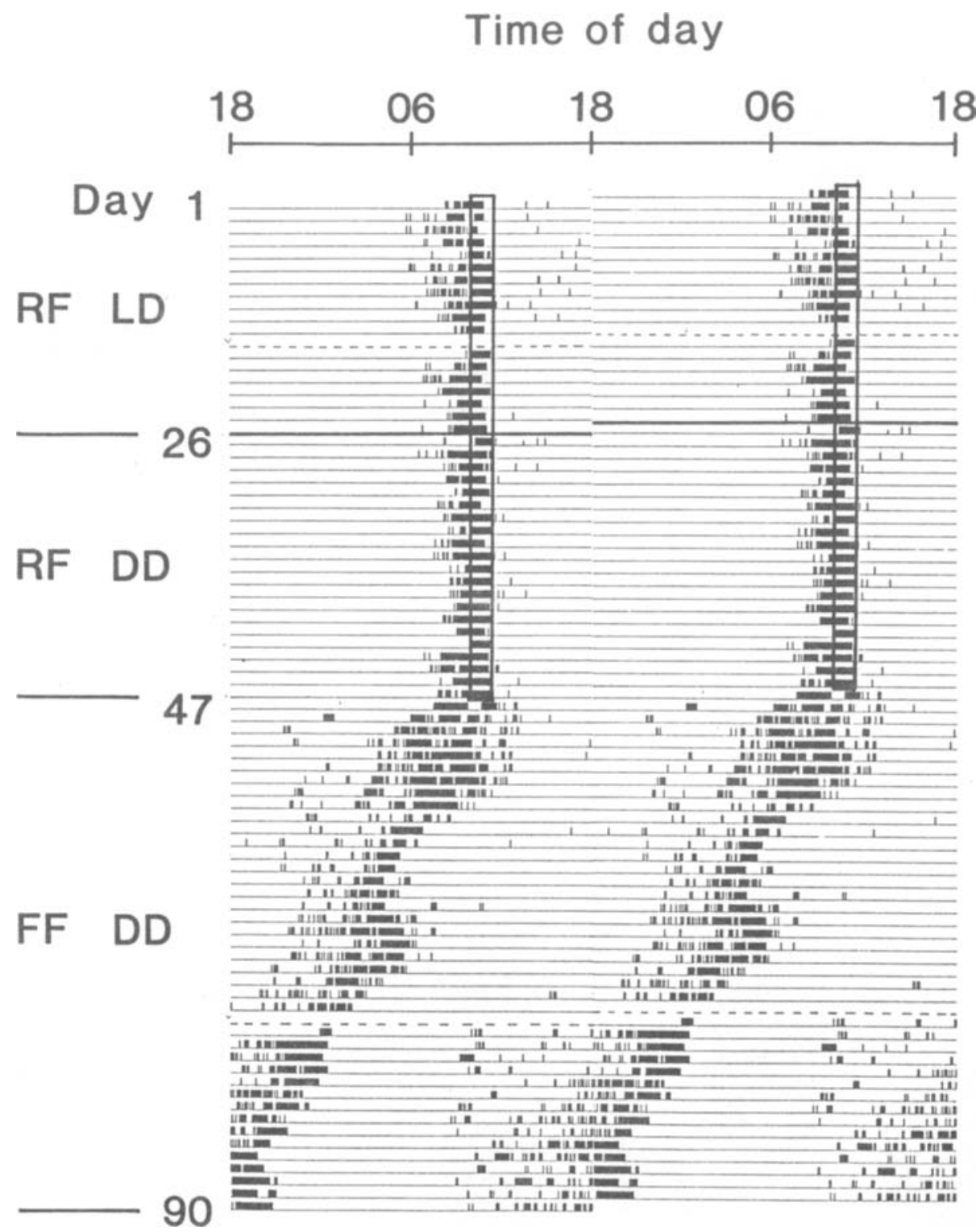

Figure 4. Temporal distribution of pecking responses for Bird P821. The broken line shows the period for which data are missing. Data for the session with the key illuminated only for the access period are not presented here. Day 1 is the day when continuous key illumination was introduced for the first time. RF = restricted food access; $F F=$ free food access.

was presented. The honeybees did not learn the food access time when the tray was presented within a cycle longer or shorter than around $24 \mathrm{~h}$. These results suggest that the bee's biological clock was involved in the learning and memory of time. The present study did not examine food-anticipatory activity under restricted food access with a noncircadian period. However, it is possible that the biological clock controls the learning process of the access period under restricted food access in the pigeon, since anticipatory pecking was observed when the bird was placed in a situation with no external cue for timing. We must examine the anticipatory activity under the noncircadian periodic feeding condition as the next step of our research.
Patterns of food-anticipatory activity differed with different lighting conditions. The effect of phase shift of the $L D$ cycle was examined with Bird P831. In RF-LD, anticipatory pecking occurred 1 or $2 \mathrm{~h}$ before the onset of the food access period. In RF-DL, pecking occurred, not prior to the food access period, but during the period. Since no cues existed for the food access period, the first 10 pecks that occurred during this period might also have been anticipatory. The bird seemed to anticipate the access period more precisely in RF-DL than under the other conditions.

The pecking that occurred in RF-DL also suggests that the activity prior to the access period might be due to the activity rhythm that is entrained to the LD cycle. It has 
been reported that the LD cycle is the most potent Zeitgeber for the pigeon's activity rhythm (Miselis \& Walcott, 1970). As was seen in FF-LD, the activity level was high throughout the light period of the cycle, but it was almost suppressed in the dark period. The LD cycle could also entrain the physiological activity. The light period might activate physiological function involved in the foodtaking mechanism. Since the basic activity level was high in the light period, food-related motivation easily increased prior to the access period when feeding was restricted. The food-anticipatory pecking thus indicated a behavioral aspect of the increased motivational state. In the dark period, pecking was not observed. Therefore, there is a possibility that the bird was not so highly motivated by the restricted access, which could also account for the fact that anticipatory pecking did not occur. However, the bird actually got access to food by showing pecking activity during the access period even in RF-DL. This suggests that the bird learned and retained the access period even if it was not so active in the dark period.

The lack of anticipation has not so far been obtained in rodents. The rat shows anticipatory wheel-running even when the access period is scheduled in the subjective night (Edmonds \& Adler, 1977; Rosenwasser et al., 1984). In the ring dove, however, the food-taking behavior itself may be skipped altogether if the access period is scheduled in the subjective night, imperiling the bird's health (M. Terman, personal communication, March 1987). Such discrepant results suggest that the degree of anticipatory behavior, and food-taking behavior, may depend on the demand requirements of the schedule.

Data obtained in FF-DD after the bird was released from RF suggest that the activity rhythms in RF-DD and RF-LL were entrained to the periodic food access. The bird was probably activated as the access period approached, and anticipatory pecking occurred. Therefore, anticipatory pecking was related to the activity level that was entrained to the LD cycle or to periodic food access in the continuous conditions.

The phenomenon similar to relative coordination was observed in the RF-DD condition. This phenomenon can usually be seen when circadian rhythm is entrained to a weak light intensity of the light-dark cycle. When a light-dark cycle is not so distinct as a Zeitgeber, the activity shows incomplete entrainment to it. The functional parallelism between food entrainment and light-dark entrainment is not yet clear. Evidence from rodents suggests that circadian anticipatory behavior is controlled by a neural timing system distinct from light-sensitive circadian activity rhythms. However, the anticipatory response observed in the present study might suggest that a foodsensitive circadian rhythm of activity is entrained to periodic food access. The response might also suggest that the system underlying food entrainment is similar to that of light-dark entrainment.

Bird P831 showed a free-running rhythm with a period shorter than $24 \mathrm{~h}$ when it was shifted from FF-LD to FF-DD, but a free-running period longer than $24 \mathrm{~h}$ when it was shifted from RF-LL to FF-DD. Bird P821 showed an unstable free-running period in FF-DD after RF. These results suggest that $\mathrm{RF}$ sessions change the free-running period. However, it also seems likely that the prolonged free-running period of Bird P831 when shifted from RF-LL would be an aftereffect of LL exposure, which is a classical finding (Pittendrigh \& Daan, 1976). In birds, LL is a profound physiological assault. It blocks pineal secretory activity, which may be involved in the avian pacemaking mechanism. It blocks retinal rhythmic functions that rely on daily light-dark cycles (but which may free-run under DD). It can induce irreversible retinal damage, and therefore reduce the amount of light that might effectively drive the internal "tau-generator" through an Aschoff's-rule mechanism. Thus it frequently renders birds arrhythmic. In view of such factors, it is indeed rather impressive that such a clean, albeit modified, free-running was obtained after LL exposure (M. Terman, personal communication, March 1987). Whether RF has a similar disturbing effect on the freerunning rhythm remains an open question.

Effects of restricted food access have been investigated with rodents; in such studies, the data are expected to test the multiple-oscillator theory of the circadian system (Boulos et al., 1980). This theory is based on findings that the food-anticipatory response occurred even after lesioning of the suprachiasmatic nucleus (SCN), which is a potent oscillator (clock) regulating the circadian rhythm of rodents (Stephan et al., 1979a, 1979b; Boulos et al., 1980). It has been suggested that birds have a multiple-oscillator system consisting of the pineal gland and the SCN (Ebihara \& Kawamura, 1981; Takahashi \& Menaker, 1982). It should be clarified how the mechanism controls the food-anticipatory activity observed under restricted food access.

\section{REFERENCES}

Adler, H. E. (1963a). Psychophysical limits of celestial navigation hypothesis. Ergebnisse der Biologie, 26, 235-252.

ADLER, H. E. (1963b). Sensory factors in migration. Animal Behavior, 11, 566-577.

Bolles, R. C., \& DELoRge, J. (1962). The rat's adjustment to a-diurnal feeding cycles. Journal of Comparative \& Physiological Psychology, $55,760-762$.

Bolles, R. C., \& Stokes, L. W. (1965). Rat's anticipation of diurnal and a-diurnal feeding. Journal of Comparative \& Physiological Psychology, 60, 290-294.

Boulos, Z., Rosenwasser, A. M., \& Terman, M. (1980). Feeding schedules and the circadian organization of behavior in the rat. $B e-$ havioral Brain Research, 1, 39-65.

Boulos, Z., \& Terman, M. (1980). Food availability and daily biological rhythms. Neuroscience \& Biobehavioral Reviews, 4, 119-131.

Ebihara, S., \& Kawamura, H. (1981). The role of pineal organ and the suprachiasmatic nucleus in the control of circadian locomotor rhythms in the Java sparrow, Padda oryzivora. Joumal of Comparative Physiology, 141, 207-214.

EDMonds, S. C., \& ADLER, N. T. (1977). The multiplicity of biological oscillators in the control of circadian running activity in the rat. Physiology \& Behavior, 18, 921-930.

MiseuIs, R., \& WALCoTt, C. (1970). Locomotor activity rhythms in homing pigeons (Columba livia). Animal Behavior, 18, 544-551.

Pittendrigh, C. S., \& DaAn, S. (1976). A functional analysis of cir- 
cadian pacemakers in nocturnal rodents: I. The stability and lability of spontaneous frequency. Journal of Comparative Physiology, 106, 223-252.

Renner, M. (1960). The contribution of the honey bee to the study of time-sense and astronomical orientation. Cold Spring Harbor Symposia on Quantitative Biology, 25, 361-367.

Richter, C. P. (1927). Animal behavior and internal drives. Quarterly Review of Biology, 2, 307-343.

Rosenwasser, A. M., Pelchat, R. J., \& Adler, N. T. (1984). Memory for feeding time: Possible dependence on coupled circadian oscillators. Physiology \& Behavior, 32, 25-30.

STEIN, H. (1951). Untersuchungen über den Zeitsinn bei Vögeln. Zeitschrift für Vergleichende Physiologie, 33, 387-403.

Stephan, F. K., Swann, J. M., \& Sisk, C. L. (1979a). Anticipation of 24-hr feeding schedules in rats with lesions of the suprachiasmatic nucleus. Behavioral \& Neural Biology, 25, 346-363.
Stephan, F. K., SwanN, J. M., \& Sisk, C. L. (1979b). Entrainment of circadian rhythms by feeding schedules in rats with suprachiasmatic lesions. Behavioral \& Neural Biology, 25, 545-554.

TAKahashi, J. S., MENaker, M. (1982). Role of the suprachiasmatic nuclei in the circadian system of the house sparrow, Passer domesticus. Joumal of Neuroscience, 2, 815-828.

Terman, M. (1983). Behavioral analysis and circadian rhythms. Advances in Analysis of Behaviour, 3, 103-141.

Terman, M., Gibbon, J., Fairhurst, S., \& Waring, A. (1984). Daily meal anticipation: Interaction of circadian and internal timing. $A n$ nals of the New York Academy of Sciences, 423, 470-514.

(Manuscript received November 17, 1986; revision accepted for publication May 1, 1987.) 